\title{
Distinct temporal recruitment of Plasmodium alveolins to the subpellicular network
}

\author{
Annie Z. Tremp • Fatimah S. Al-Khattaf • \\ Johannes T. Dessens
}

Received: 30 July 2014 / Accepted: 25 August 2014 / Published online: 4 September 2014

(C) The Author(s) 2014. This article is published with open access at Springerlink.com

\begin{abstract}
The zoite stages of malaria parasites (merozoite, ookinete and sporozoite) possess a distinctive cortical structure termed the pellicle, which is defined by a double membrane layer named the inner membrane complex (IMC). The IMC is supported by a cytoskeleton of intermediate filaments, termed the subpellicular network (SPN). Plasmodium IMC1 proteins, or alveolins, make up a conserved family of structurally related proteins that comprise building blocks of the SPN. Here, using green fluorescent protein (GFP) tagging in P. berghei, we show that the alveolins $P b \mathrm{IMCl}$ and $P b \mathrm{IMCle}$ are expressed in all three zoite stages. Our data reveal that $P b I M C 1$ e is assembled into the SPN concurrent with pellicle development, while $P b \mathrm{IMCl}$ is assembled after pellicle formation. In the sexual stages, these processes are accompanied by different gene expressions from maternal and paternal alleles: $P b I M C l e$ is expressed uniquely from the maternal allele, while $P b I M C 1 c$ is expressed from the maternal allele in gametocytes, but from both parental alleles during ookinete development. These findings establish biogenesis of the cortical cytoskeleton in Plasmodium to be a complex and dynamic process, involving distinct parental gene expression and chronological recruitment of its protein constituents. While allelic replacement of the pbimclc and pbimcle genes with GFP-tagged versions was readily achieved using double crossover homologous recombination, attempts to disrupt these genes by this strategy only resulted in the integration of the selectable marker and GFP
\end{abstract}

The authors Annie Z. Tremp and Fatimah S. Al-Khattaf contributed equally to this work.

A. Z. Tremp • F. S. Al-Khattaf • J. T. Dessens $(\triangle)$

Pathogen Molecular Biology Department, Faculty of Infectious and

Tropical Diseases, London School of Hygiene and Tropical

Medicine, Keppel Street, London WC1E 7HT, UK

e-mail: johannes.dessens@1shtm.ac.uk

\section{F. S. Al-Khattaf}

Department of Infection Control, College of Medicine, King Saud University, Riyadh, Saudi Arabia reporter into non-specific genomic locations. The recurrent inability to disrupt these genes provides the first genetic evidence that alveolins are necessary for asexual blood-stage parasite development in Plasmodium.

Keywords Plasmodium berghei · Cytoskeleton . Intermediate filament $\cdot$ Sexual stages $\cdot$ Sporogonic development

\section{Introduction}

Malaria parasite transmission is initiated by the ingestion of gametocytemic blood by a vector mosquito, which initiates gametogenesis followed by fertilization. Zygotes transform into motile ookinetes that traverse the gut wall of the insect and transform into oocysts (Meis \& Ponnudurai, 1987; Meis et al., 1989). An approximately 2 -week period of growth and replication culminates in hundreds of motile sporozoites being released from each oocyst. These invade the salivary glands and are transmitted to new hosts, again by blood feeding of the insect. Once in the host, sporozoites rapidly infect liver cells and replicate each to produce thousands of merozoites. The motile merozoites are released into the bloodstream, where they infect red blood cells and either replicate to form more merozoites or differentiate into sexual-stage male and female gametocytes to complete the life cycle.

The three motile and invasive stages (zoites) of Plasmodium species (i.e. ookinetes, sporozoites and merozoites), as well as zoites of other apicomplexan parasites, possess a similar cortical structure termed the pellicle. The pellicle is essentially made up of the plasma membrane and an underlying double membrane structure termed the inner membrane complex (IMC) (Bannister et al., 2000; Morrissette \& Sibley, 2002; Santos et al., 2009). Closely associated with the IMC on its cytoplasmic side is a network of intermediate filaments 
termed the subpellicular network (SPN), which supports the pellicular membranes and provides mechanical strength to the cell (Mann \& Beckers, 2001). The pellicular membranes are further supported by subpellicular microtubules that run lengthwise from the anterior towards the posterior end, completing the cortical cytoskeleton (Bannister et al., 2000; Morrissette \& Sibley, 2002; Santos et al., 2009).

Several members of an Apicomplexa-specific family of proteins termed IMC1 proteins have been identified as components of the SPN (Khater et al., 2004; Mann \& Beckers, 2001). Structurally related proteins from ciliates and dinoflagellate algae have since been added to this protein family renamed 'alveolins', which now defines the Alveolata infrakingdom (Gould et al., 2008). In the genus Plasmodium, the number of members of the alveolin family has risen to 12 (Kono et al., 2012), which are encoded by conserved and syntenic genes. The alveolin family members display differential expression between the three zoite stages of the parasite, with the largest repertoires present in the ookinete and sporozoite according to proteomic studies (Florens et al., 2002; Hall et al., 2005; Lasonder et al., 2002; Lindner et al., 2013; Treeck et al., 2011). It has been shown in the rodent malaria species Plasmodium berghei that the disruption of individual alveolin family members expressed in sporozoites ( $P b I M C 1 a)$, in ookinetes $(P b I M C 1 b)$ or in both these zoites $(P b I M C 1 h)$ results in morphological abnormalities that are accompanied by reduced tensile strength of the zoite stages in which they are expressed (Khater et al., 2004; Tremp \& Dessens, 2011; Tremp et al., 2008; Volkmann et al., 2012). Besides roles in morphogenesis and mechanical strength, the Plasmodium alveolins are also involved in gliding motility in both ookinetes and sporozoites, most likely through interactions with components of the glideosome that are situated within the pellicular cytoplasm (Khater et al., 2004; Tremp \& Dessens, 2011; Tremp et al., 2008; Volkmann et al., 2012).

In this study, we investigate the expression, subcellular distribution and function of two further members of the alveolin/IMC1 protein family, $P b \mathrm{IMC} 1 \mathrm{c}$ and $P b \mathrm{IMC} 1 \mathrm{e}$, revealing fundamental differences in the manner they are expressed and participate in zoite morphogenesis. In addition, we provide the first evidence that both $P b I M C 1 \mathrm{c}$ and $P b I M C 1$ e are essential for the development of the asexual blood stages of the parasite in the host, underpinning the alveolins as potential target molecules for chemotherapybased intervention.

\section{Materials and methods}

Animal use

All laboratory animal work undergoes regular ethical review by the London School of Hygiene and Tropical Medicine and has been approved by the United Kingdom Home Office. Work was carried out in accordance with the United Kingdom Animals (Scientific Procedures) Act 1986 implementing European Directive 2010/63 for the protection of animals used for experimental purposes. Experiments were conducted in 6-8-week-old female CD1 mice, specific pathogen free and maintained in filter cages. Animal welfare was assessed daily, and animals were humanely killed upon reaching experimental or humane endpoints. Mice were infected with parasites suspended in RPMI or PBS by intraperitoneal injection or by infected mosquito bite on anaesthetized animals. Parasitemia was monitored regularly by collecting a small drop of blood from a superficial tail vein. Drugs were administered by intraperitoneal injection or, where possible, supplied in drinking water. Parasitized blood was harvested by cardiac bleed under general anaesthesia without recovery.

Parasite maintenance, transmission, culture and purification

P. berghei ANKA clone 234 parasites were maintained as cryopreserved stabilates or by mechanical blood passage and regular mosquito transmission. Ookinete cultures were set up overnight from gametocytemic blood as previously described (Arai et al., 2001). After 18-20 h, ookinetes were purified via ice-cold $0.17 \mathrm{M}$ ammonium chloride lysis and centrifugation at $800 \times \mathrm{g}$ for $10 \mathrm{~min}$, followed by PBS washes. Mosquito infection and transmission assays were previously described using Anopheles stephensi (Dessens et al., 1999; Khater et al., 2004), and infected insects were maintained at $20{ }^{\circ} \mathrm{C}$ at approximately $70 \%$ relative humidity.

\section{Gene-targeting constructs}

The entire pbimclc coding sequence plus ca. $0.55 \mathrm{~kb}$ of upstream sequence was PCR amplified from $P$. berghei genomic DNA with primers pDNR-IMC1c-F (ACGAAGTTATCA GTCGACGGTACCAAGTGCATTTAGTATGTTGTGGC) and pDNR-IMC1c-R (ATGAGGGCCCCTAAGCTTCTGC ATGTACCTGTACAGCAT) and cloned into SalI/HindIIIdigested pDNR-EGFP (Tremp et al., 2008) by in-fusion cloning to give plasmid pDNR-IMC1c/GFP. The 3'UTR of pbimclc was amplified with primers pLP-IMC1c-F (ATAT GCTAGAGCGGCCTTTCGTGAAAAATGCAGTTAACA) and pLP-IMC1c-R (CACCGCGGTGGCGGCCGAAAGA AGACAATAAATAAAATAGAAAGTATGG) and the resulting ca. $0.6 \mathrm{~kb}$ fragment cloned into NotI-digested pLPhDHFR by in-fusion cloning to give plasmid pLP-hDHFR/ IMC1c. The pbimclc/gfp-specific sequence from pDNRIMC1c/GFP was transferred to pLP-hDHFR/IMC1c by Cre/ loxP recombination to give the final construct pLP-IMC1c/ GFP. This plasmid served as template in a PCR-based sitedirected mutagenesis using primers IMC1c-KO-F (CAACCG TCATGAGTAAAGGAGAAGAACTTTTCAC) and IMC1c- 
KO-R (TTACTCATGACGGTTGATGTCTCTTTAGTGT). The resulting PCR product was circularized using in-fusion to give plasmid pLP-IMC1c-KO. In this plasmid, the pbimclc coding sequence except for the first amino acids has been removed.

The entire pbimcle coding sequence plus ca. $0.58 \mathrm{~kb}$ of upstream sequence was PCR amplified from genomic DNA with primers pDNR-IMC1e-F (ACGAAGTTATCAGTCG ACGGTACCGCATAAATTAACTTAGTTTCATTGAACT TC) and pDNR-IMC1e-R (ATGAGGGCCCCTAAGCTTTC GTTTAAGACGGGTGGTAC) and cloned into SalI/HindIIIdigested pDNR-EGFP by in-fusion cloning to give plasmid pDNR-IMC1e/GFP. The 3'UTR of pbimcle was amplified with primers pLP-IMC1e-F (ATATGCTAGAGCGGCCTT TGGCTTCGATTTTTGTG) and pLP-IMC1e-R (CACCGC GGTGGCGGCCTAACAGCATTATGAAAGATTGGC) and the resulting ca. $0.87 \mathrm{~kb}$ fragment cloned into NotI-digested pLP-hDHFR by in-fusion cloning to give plasmid pLPhDHFR/IMC1e. The pbimcle/gfp-specific sequence from pDNR-IMC1e/GFP was transferred to pLP-hDHFR/IMC1e by $\mathrm{Cre} /$ loxP recombination to give the final construct $\mathrm{pLP}-$ IMC1e/GFP. This plasmid served as template in a PCR-based site-directed mutagenesis using primers IMC1e-KO-F (AATA TGTGATGAGTAAAGGAGAAGAACTTTTCAC) and IMC1e-KO-R (TTACTCATCACATATTTAGTGCCACAAT TGC). The resulting PCR product was circularized using infusion to give plasmid pLP-IMCle-KO. In this plasmid, the pbimcle coding sequence except for the first amino acids has been removed.

To generate a mCherry-tagged version in $P b \mathrm{IMClc}$, the mCherry coding sequence was amplified from pDNRmCherry/PbSR/EGFP (Carter et al., 2008) with primers pDNR-mCherry-F (CAGTCGACTTAAGCTTAGGGGCCC TCATGGTGAGCAAGGGCG) and pDNR-mCherry-R (AACGGGATCTTCTAGTTACTTGTACAGCTCGTCCAT GC) and introduced into HindIII/XbaI-digested pDNR-EGFP by in-fusion to give plasmid pDNR-mCherry. A $3.8-\mathrm{kb}$ fragment corresponding to the entire pbimclc gene plus upstream intergenic region was PCR amplified from $P$. berghei gDNA using primers IMC1c-mCherry-F (ACGAAGTTATCAGT CGAGGTACCTTCTCATTGTCAATGGCTCC) and pDNR-imc1c-R and introduced into SalI/HindIII-digested pDNR-mCherry by in-fusion to give plasmid pDNR-IMC1c/ mCherry. The $P b I M C 1 \mathrm{c} / \mathrm{mCherry}$-specific sequence from pDNR-IMC1c/mCherry was introduced into plasmid pLPhDHFR/IMC1c by Cre/lox recombination to give plasmid pLP-IMC1c/mCherry/hDHFR.

Generation and genomic analysis of genetically modified parasites

Parasite transfection, pyrimethamine selection and dilution cloning were performed as previously described (Waters et al., 1997). Prior to performing transfections, plasmid DNA was digested with $K p n I$ and SacII to remove the plasmid backbone. Genomic DNA extraction was performed as previously described (Dessens et al., 1999). For the FP-tagged lines, confirmation of correct targeting and integration into the pbimclc and pbimcle loci was carried out with diagnostic PCR across the integration sites using primer pair hDHFR/ERI-F (ACAAAGAATTCATGGTTG GTTCGCTAAACT) and IMC1c-3'R (TTAGAGCCGATT TTATCTTGTTACAC) for parasite lines IMC1c/GFP and IMC1c/mCherry; and hDHFR/ERI-F and IMC1e-3'R (AAGGTATAAAGTTTATGCATTTTAGCTATC) for parasite line IMC1e/GFP. Confirmation of the absence of the WT allele in the transgenic lines was carried out with primer pairs pDNR-IMC1c-F and IMC1c-3'R (for IMC1c/ GFP); IMC1c-5'F (CTATACCACGCAGCAACAATG) and IMC1c-3'R (for IMC1c/mCherry); and pDNR-IMCle-F and IMCle-3'R (for IMCle/GFP).

Western blot analysis

Parasite samples were heated directly in SDS-PAGE loading buffer at $70{ }^{\circ} \mathrm{C}$ for $10 \mathrm{~min}$. Proteins were fractionated by electrophoresis through NuPage 4-12 \% Bis-Tris precast gels (Invitrogen) and transferred to PVDF membrane (Invitrogen) according to the manufacturer's instructions. Membranes were blocked for non-specific binding in PBS supplemented with $0.1 \%$ Tween 20 and $5 \%$ skimmed milk for $1 \mathrm{~h}$ at room temperature. Goat polyclonal antibody to green fluorescent protein (GFP) conjugated to horse radish peroxidase (Abcam ab6663) diluted 1:5,000 was applied to the membrane for $1 \mathrm{~h}$ at room temperature. After washing, signal was detected by chemiluminescence (Pierce ECL western blotting substrate) according to the manufacturer's instructions.

\section{Microscopy}

For assessment of fluorescence, live parasite samples were assessed, and images captured, on a Zeiss LSM510 inverted confocal microscope or on a Zeiss Axioplan-2 fluorescent microscope with Retiga 2000R CCD camera system and Volocity software.

\section{Results}

Structure of the Plasmodium alveolins IMC1c and IMC1e

The Plasmodium IMC1 protein family was first published in 2004 using gene models of $P$. yoelii (Khater et al., 2004). PbIMC1c (PBANKA_120200) is composed of 278 amino acids encoded by a single exon. PbIMC1c and its orthologous 
proteins share a highly conserved amino-terminal domain related to the IMCp domain superfamily (Pfam12314) that defines the IMC1 proteins/alveolins (Fig. 1a). The proteins also possess a conserved cysteine motif at the carboxyterminus similar to the cysteine motifs described in PbIMC1a and TgIMC1 (Fig. 1a) that is believed to act as a palmitoylation signal (Khater et al., 2004; Mann \& Beckers, 2001). PbIMC1e (PBANKA_040270) is composed of 512 amino acids encoded by a single exon. Sequence conservation is limited to an IMCp domain in their central portions
(Fig. 1b). Interestingly, the Plasmodium imcle locus is located directly downstream of its family member imcla in the opposite orientation, suggesting that these two genes could be sharing promoter elements.

Fluorescent protein tagging of PbIMC1c and PbIMC1e

To achieve GFP tagging of PbIMC1c and PbIMC1e, we adopted a strategy of double crossover homologous recombination in which the wild-type alleles were replaced with

\begin{abstract}
A
Pb MDNSHLSRNSYQKIDNIDTKETSTVDRKWVALTAYQPVDVVTKTVEVPVIKTVEKFVPKTI IQEKIIHVPKNVTH IVEKIVEVPEVKY IEKVVEVPH Pf MADSIKSSNSFQKLDNIDAKETSTVDRKWVALTAYQPVDVVTKTVEVPIIKTVEKYVPKTIIQEKIIHVPKNVTHIVEKIVEVPEVKY IEKIVEVPH PV MSGSVNRSNSFQKADSMDAKETSTVDRKWVALTAYQPVDVVTKTVEVPVIKTVEKFVPKTI IQEK I IHVPKNVTH IVEK IVEVPEVKY IEKIVEVPH Pk MEGSIKNSNSFQKADCMDAKETSTVDRKWVALTAYQPVDVVTKTVEVPVIKTVEKFVPKTI IQEK I IHVPKNVTH IVEK IVEVPEVKY IEKIVEVPH

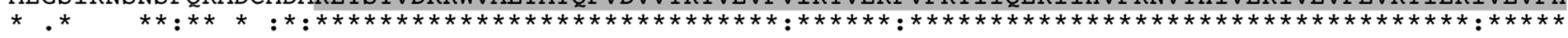

Pb IHYKNKYVPKIEVVEKIVERQKIIEKWHDKIVEVPQIKEVVRYKEIEDTEEVIKYVPRDSKN INWEEEYKKYTEGKAG-KFSL-NNTYQQQMNSYNQ Pf IHYKNKYVPKIEIVEKVVERQKI IEKWHDKIVEVPQIKEVVRFKQIEDSEEI IKYVPRNSKN IDWEDEYKKYTESKGLQRYSLDQNNIYQQANSFNQ PV IHYKNKYVPKIEVVEKVVEROKIIEKWHDKIVEVPOIKEVVRFKEIEDAEEVIKYIPRNSKNINWEEEYOKYTORKGOERYSLDNNAYOOKMSSYND Pk IHYKNKYVPKIEIVEKVVERQKI IEKWHDKIVEVPQIKEVVRFKEIEDAEEVIKYIPRNSKNINWEEEYQKYTQRKGQERYSLDNNNYQQKMNSYND

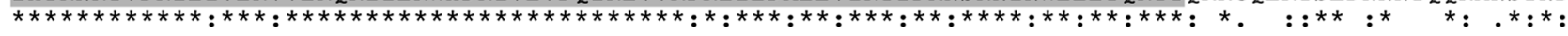

$\mathrm{Pb}$ INENAYARNSHSRS INVMN--SKTANDQTNMRSEDFSQINFFNQYNGE---SMDQH-IMMPSSSFNMNGSFQFKRLPSEEAKPVGCCTGTCR Pf FNENAYNQNAFNRSYELLNKQSSVKS-QNNASGENFSQMNFYNQYSGAN----FEQERS IQASNFEPSGSMQLKRLSSEE IKPAGCC SAACT PV YNEKAYSQNAYSRSLDFMNKQSSSLNNQSNIRSEDFSQINFFNQYIGSAGASYDKQRNSVPTSSFDARGSLQLKRMPSEEAKPVGCCTSACT Pk YNEKAYSQNAYSRSLDFMNKQSSSLNNQSNIRSEDFSQINFFNQYSGSGGASYEQHRNSIPTSNFEALGSLQMKRLPSEEAKPVGCCTSACN

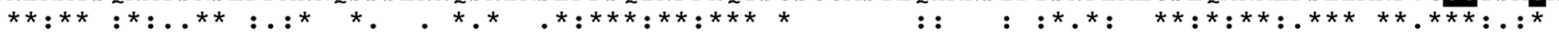

B

Pb MKNNCGE-GSTYNNQGYKFCETSNCG---TKYVDQNGK-TKSQGDQQ--YNIMDNIFLKESNNGNDNNY--------------------------LD Pf MVKCNDS-----NQ-SKNCTRNFCN---VNEEDRKEEGMMSQKDNENMYNPND-IHNNNYNNYYTNNYSVEKNK------FSQNSMNQENYENFVD PV MLNNCGE-----Pk MLNNCGE---------------SNCRGYAQNLCDQQGKKGTMQRDPNDQYYQVASNLFSNGDGSDRREG---------------SSMGGTRVLNSGD *

: $\quad$ : : : $: *$ * $\quad$ * $\quad$. : . :
\end{abstract}

$\mathrm{Pb}$ KVPENARMLKPLVQEKIIEIMKPEIEEKIIEVPQIQYVEKLVEVPHVILQEKLIHVPKPVIHERIKKCPKTIFOEKIVEVPQIKIVDKIIEVPQYVY Pf CPPQNARILKPLIQEKIVE IMKPEIEEKI IEVPQVQY IEKLVEVPHVILQEKLIH I RKPVIHERIKKCSKTIFQEKIVEVPQIKVVDKIVEVPQYVY PV SPPQNARILKPLVQEKVVE IMKPE IEEKI IEVPQVQY IEKLVEVPHVILQEKLIHVPKPVIHERIKKCPKTIFQEKIVEVPQIKVVDKIVEVPQYVY Pk APPQNARILKPLVQEKVVEIMKPEIEEKIIEVPQVQY IEKLVEVPHVILQEKL IHVPKPVIHERIKKCPKTIFQEKIVEVPQIKVVDKIVEVPQYVY

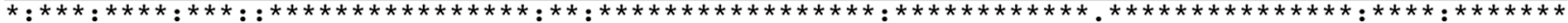

$\mathrm{Pb}$ OEKIIOVPKVMVOERIIPVPKKVIKEKIVEIPOIELKNINIEKIEEIPEYIPEVVKRDVPYTOIIDRPFHVEKIVEVPHVOHIYRNIVSPOYRHIPK Pf OEKIIEVPKIMVQERIIPVPKKIVKEKIVEIPQIELKNIDIEKVQEIPEYIPEVVQKDIPYTQIVDRPYHVEKIVEVPHVQHIYRNIVSPQYRHIPK PV QEKIIQVPKIMVQERIIPVPKKVIQEKIVEIPQIELKNINIEKVQEIPEYIPEVVKKDIPYTQIVDRPFHVEKIVEVPHVQHIYRNIVSPQYRHIPK $\mathrm{Pk}$ QEKIIQVPKIMVQERIIPVPKKVIQEKIVEIPQIELKNINIEKVQEIPEYIPEVVKKDIPYTQIVDRPFHVEKIVEVPHVQHIYRNIVSPQYRHIPK

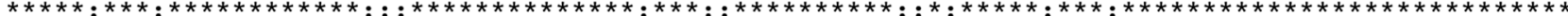

Pb PVEVPMAHYRTFPVEKLVDRNVPVPVEIQIVQEFLCPKIEARYKEIPVPVHVQRIIEHPIPKDAMNNPFLLPLYYQEDNFNT--SKNSTVNGSNTPPf PVEIPMAHYRTFPVEKIVDRNVPVPVELQIVQEFLCPKIEARYKEIPVPVHVQRIIEHPIPKDAMNNPHLLPLYYQEDNN----IEMST-NKKNTME PV PVEVPMAHYRTFPIEKLVDRNVPVPVELQIVQEFLCPKIEARYKEIPDPVHVQRIIEHPIPKDAMNNPFLLPLYYQEDNIDTASTKGSS--------Pk PVEVPMAHYRTFPIEKLVDRNVPVPVELQIVQEFLCPKIEARYKEIPVPVHVQRIIEHPIPKDAMNNPFLLPLYYQEDNIDTTSSKGSS---------

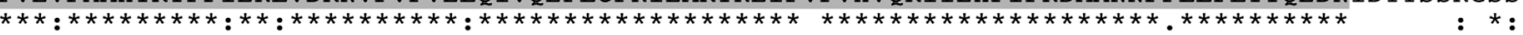

$\mathrm{Pb}$ QN-NKGCFMFNWNKNHNQKTMKSRNN-----SVELLLHNDNKNNEFFNINEANINPNINLKTSINQYSGNTFRDINNIPMDANIMNMHALGQCQS IN Pf QNKNKNCFIFNLNRND------SRNSSHASPSVELLLHNDKSQKQFYNN--------HTNKKTSIISNP-NVFNNYN-----------ONGTLNQFN PV ----KNCF SFNWNNKQN---ATKINKGSNQPSVELLLHNDKHHS-KVNPNQDQHMRAKFVSPSESVLHTTHLRDDD--PLNAQPTYHYPNGTMHNGA Pk ----KNCF PFNWNNKQN---ATK INKGTNHQSVELLLHNDKYHN-KVNINHDANMRAKFVPPSGNVYPSTHLRDDN--PLTAQSTYHYPNGTQH ISA

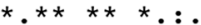
. *
$* * * * * * * * *: \quad:$.
*
.* $\quad$ * : : :

$\mathrm{Pb}$ PID---NSNNQYNFIHNPAAMNYSS-PNFNS------------EIPYNPNASLKISNSMSYPMESIPLKKPYPIVSHIVPPVLNE

Pf PND---NINNNMEHIIHHNNNNIPSNVNFKSFHDNVITHNK INNNMPYENNTVLKNNYSVNIPOTYTTKEFANNIYSSPSP-VLSSSHTEHA

PV SIHGNAALNQMYRTQHVTSQAAYP SNFNTAMLS--------PQDQNDMFNGSNSMGFTHPCDSELG--GAYPPISALGQVSPHGELN

Pk PLHGNASLNHLYKAQHVNSHTAYPSNFNTAMLS------------PQDQNDMFNGSNSMGFTHPSDTEMG--NAYSPISALGQVSPHSELN

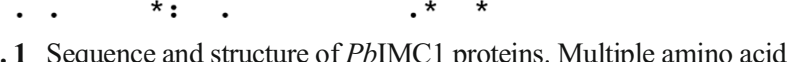

Fig. 1 Sequence and structure of $P b \mathrm{IMC} 1$ proteins. Multiple amino acid
sequence alignment of the predicted IMC1c (a) and IMC1e (b) proteins from P. berghei $(\mathrm{Pb})$, P. knowlesi $(\mathrm{Pk})$, P. vivax $(\mathrm{Pv})$ and P. falciparum (Pf). Indicated are conserved alveolin domains (shaded) and gaps introduced to allow optimal alignment (hyphens). Conserved amino acid identities (asterisks) and similarities (colons and points) are indicated underneath. Also shown is a conserved cysteine motif at the carboxyterminus of IMC1c (black shading). The alignment was made with ClustalW 
recombinant full-length wild-type alleles fused to GFP at their carboxy-terminus (Fig. 2a). After the transfection of purified schizonts, pyrimethamine-resistant parasites were selected and cloned by limiting dilution as described (Tremp \& Dessens, 2011; Tremp et al., 2008) to give parasite lines IMC1c/GFP and IMC1e/GFP, respectively. PCR diagnostic for integration into the pbimclc locus produced a specific band of $1.8 \mathrm{~kb}$ in the IMC1c/GFP clones, while PCR diagnostic for the presence of the wild-type imclc allele gave a specific band of $2.1 \mathrm{~kb}$ only in wild-type parasites (Fig. 2b). Likewise, PCR diagnostic for integration into the pbimcle locus produced a specific band of $2.0 \mathrm{~kb}$ in the IMCle/GFP clones, while PCR diagnostic for the presence of the wild-type pbimcle allele gave a specific band of $3.0 \mathrm{~kb}$ only in wild-type parasites (Fig. 2b). Both genetically modified parasite lines generated displayed normal parasite development in mouse and mosquito and were readily transmitted by sporozoiteinfected mosquito bites, indicating that the carboxy-terminal GFP fusions had not adversely affected the function of $P b I M C 1 c$ and PbIMCle. Both parasite lines displayed GFP fluorescence in ookinetes (see below), and immuno blot analysis of purified, cultured ookinetes with anti-GFP antibodies detected specific bands corresponding to the $P b \mathrm{IMC} 1 \mathrm{c}$ and $P b I M C 1 e$ fusion proteins with GFP, respectively (Fig. 2c).

\section{Life-stage expression of PbIMC1c and PbIMC1e}

The expression and subcellular distribution of $P b \mathrm{IMC} 1 \mathrm{c}$ and $P b I M C 1$ e were assessed by UV and laser scanning microscopy of live parasites. IMC1c/GFP parasites displayed strong fluorescence throughout asexual blood-stage development that appeared cytoplasmic, except in mature schizonts where it showed clear peripheral localization in individual merozoites (Fig. 3). To assess PbIMC1c expression in the mosquito stages, we set up ookinete cultures and infected A. stephensi vector mosquitoes. Cultured ookinetes displayed very strong fluorescence with a cortical distribution (Fig. 3). Sporulated oocysts and sporozoites also displayed strong fluorescence, which was concentrated at the cortex of the sporozoites (Fig. 3). These combined observations are fully consistent with a pellicular localization of $P b I M C 1 \mathrm{c}$ and are in agreement with it being a predicted SPN resident. Besides the peripheral distribution of $P b \mathrm{IMCl}$ in sporozoites, a thickened area was present near one extremity of the cell (Fig. 3). In $P$. berghei sporozoites, the nucleus is consistently positioned closer to the posterior end of the cell (Kudryashev et al., 2010). Accordingly, based on its position relative to the sporozoite nucleus, as well as its localization away from the sporoblast in sporulated oocysts (Fig. 3), the discrete area of fluorescence appears to be located at the anterior end.

In contrast to IMC1c/GFP parasites, IMC1e/GFP parasites exhibited very weak GFP-based fluorescence in blood stages that required recording with a $\mathrm{CCD}$ digital microscope camera (Fig. 4). Because of these low fluorescence levels, it was difficult to discern a specific subcellular distribution. In contrast to the blood stages, mature ookinetes displayed much stronger GFP fluorescence that was distributed predominantly in the cell cortex (Fig. 4), consistent with a pellicular
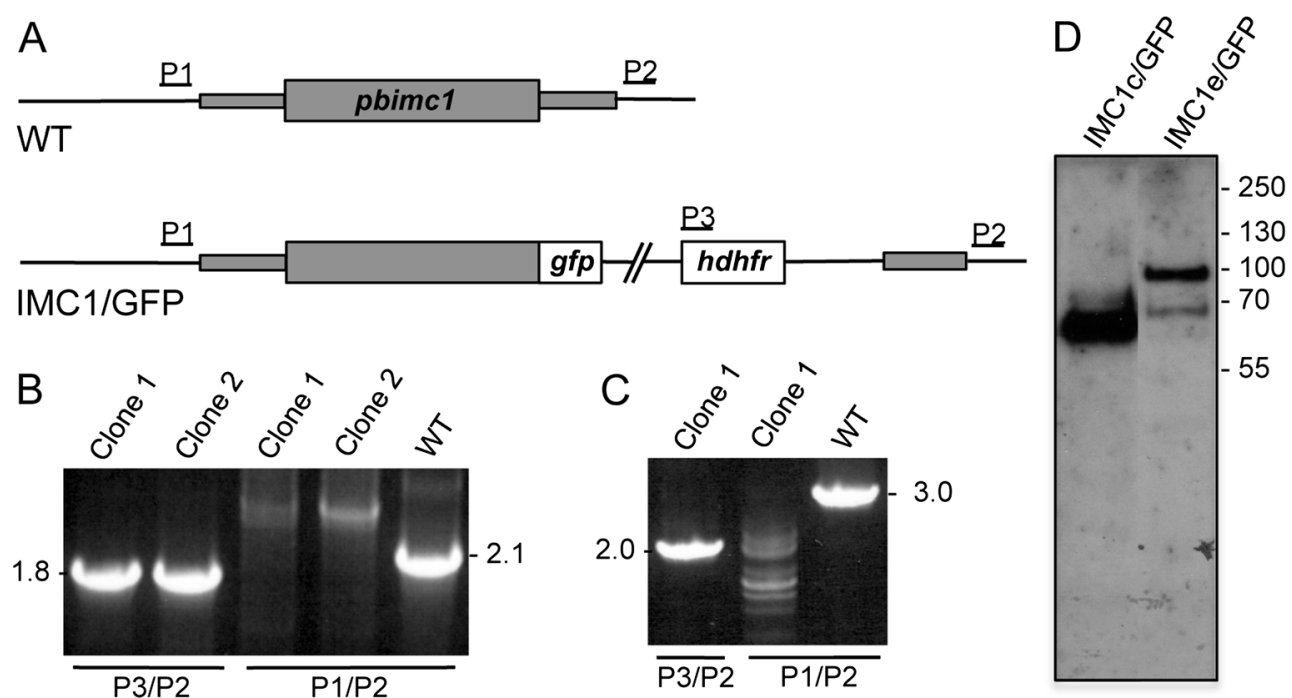

Fig. 2 Generation and molecular analyses of genetically modified parasite lines. a General targeting strategy for the GFP tagging of pbimclc and pbimcle via double crossover homologous recombination. Both the wildtype (WT) and modified, GFP-tagged (IMC1/GFP) alleles are shown. The pbimcl gene is indicated with coding sequence (wide bars) and noncoding sequence (narrow bars). Also indicated are the enhanced GFP module $(g f p)$, the hDHFR selectable marker gene cassette $(h d h f r)$ and primers used for diagnostic PCR amplification (P1-P3). b PCR diagnostic

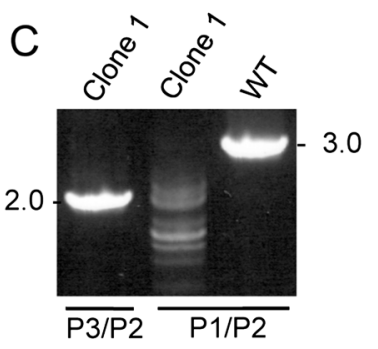

for the presence of the GFP-tagged pbimcl alleles using primers $\mathrm{P} 2$ and P3 (P3/P2) and the absence of the wild-type pbimcl alleles using primers $\mathrm{P} 1$ and $\mathrm{P} 2$ (P1/P2) from clonal parasite populations of IMC1c/GFP (left panel) and IMC1e/GFP (right panel). WT parasites are included as positive controls for the unmodified alleles. $\mathbf{c}$ Western blot analysis of purified, cultured ookinete samples of parasite lines IMC1c/GFP and IMC1e/GFP using anti-GFP antibodies, showing corresponding $\mathrm{Pb}$ IMC1::GFP fusion proteins 


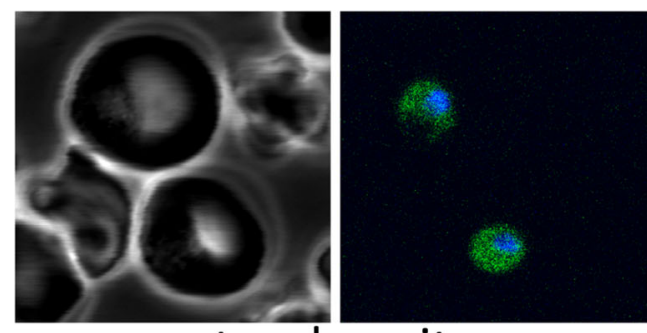

trophozoite

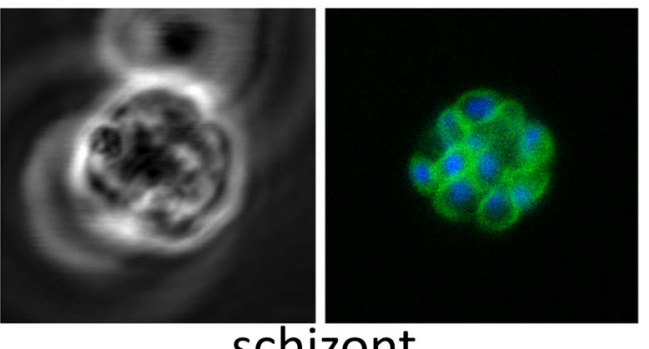

schizont

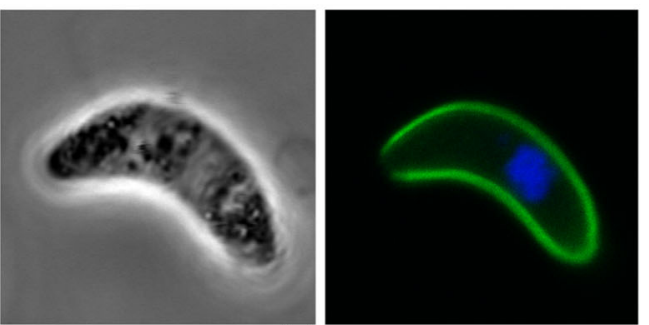

ookinete

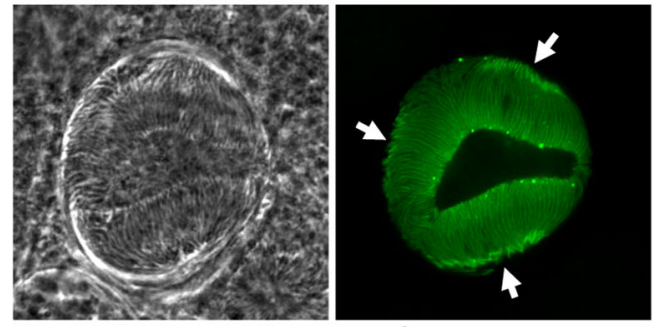

oocyst

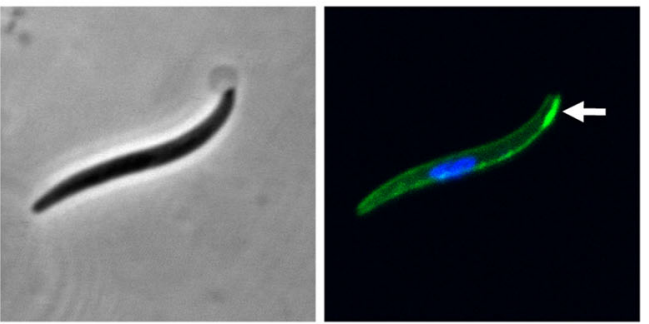

sporozoite

Fig. 3 Expression and subcellular localization of PbIMC1c. Confocal bright-field and GFP fluorescence images of trophozoite, schizont, ookinete, mature oocyst and sporozoite life stages. Hoechst DNA staining (blue) indicates position of nuclei. Arrows point to anterior structures in sporozoites

localization of the protein and its predicted function in the SPN. PbIMCle was also present in an unknown structure situated at one extremity of the ookinete (Fig. 4). A genetic cross with parasite line G2/GFP, which labels the collar (i.e. an
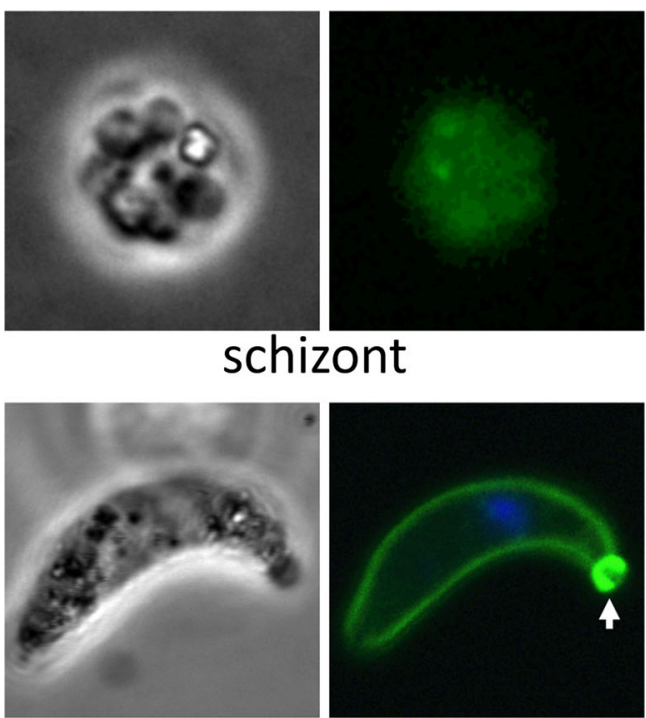

ookinete

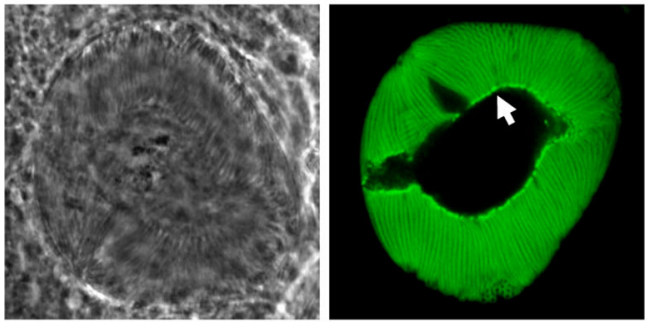

oocyst

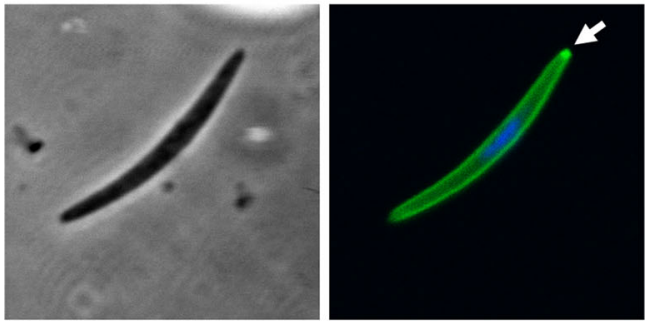

sporozoite

Fig. 4 Expression and subcellular localization of $P b I M C 1$ e. Bright-field and GFP fluorescence images of schizont, ookinete, mature oocyst and sporozoite life stages. The schizont image was captured using a CCD camera due to the low levels of fluorescence, while the other images were captured by confocal microscopy. Hoechst DNA staining (blue) indicates the position of nuclei. Arrows point to posterior structures in ookinetes and sporozoites

apical cap-like structure of the ookinete) (Tremp et al., 2013), gave rise to heterokaryotic ookinetes that simultaneously displayed both the PbG2-labelled collar and PbIMC1e-labelled structure (data not shown), indicating that the latter is positioned at the posterior end of the ookinete. Sporulated oocysts and sporozoites also displayed GFP florescence, which localized to the periphery of the sporozoites (Fig. 4). Sporozoites possessed a small fluorescent spot at one extremity which, based on its position relative to the sporozoites nucleus, as well as its localization in sporulated oocysts lining 
the sporoblast (Fig. 4), appears to correspond to the sporozoite posterior end.

PbIMC1c and PbIMC1e display distinct temporal recruitment to the SPN

To further study the recruitment of $P b I M C 1 \mathrm{c}$ and $P b I M C 1$ e to the pellicle, we examined retorts (i.e. immature ookinetes), which contain an elongated 'ookinete' portion that contains pellicle and a spherical 'zygote' part that does not. This revealed a marked difference between the two alveolins: whereas $P b I M C 1$ e was clearly localized to the periphery of the elongated 'ookinete' portion of the retort, $P b \mathrm{IMCl} c$ was not (Fig. 5a). In fact, $P b I M C 1$ e was detected in the pellicle of very young retorts, indicating that it is assembled into the ookinete SPN from the start of pellicle/SPN formation (Fig. 5b). Interestingly, this process was accompanied by the formation of several fluorescent spots in the spherical 'zygote' section (Fig. 5c).

When examining oocysts on IMC1c/GFP parasite-infected A. stephensi midguts at 2 weeks post-infection, we observed fully sporulated oocysts with very strong GFP fluorescence that clearly was localized at the sporozoites' cortex (Fig. 5d). However, on the same midguts, we found sporozoitecontaining oocysts that exhibited very low, baseline GFP fluorescence levels similar to not yet sporulated oocysts (Fig. 5d). These observations indicate that PbIMC1c is predominantly expressed after sporozoite budding and - similar to the situation in the ookinete-is recruited to the SPN after pellicle formation. By contrast, IMC1e/GFP parasite-infected midguts had sporulated oocysts that exhibited strong fluorescence without exception.

$\mathrm{PbIMC1}$ and $\mathrm{PbIMC1e}$ are differentially expressed from maternal and paternal alleles in the sexual stages

Interestingly, we found a weak cytoplasmic PbIMC1c::GFP expression in gametocytes, but only in females (possessing the smaller nucleus) (Fig. 6a). We did not see a discernible increase in fluorescence until some $7-8 \mathrm{~h}$ post-gametogenesis, resulting in mature ookinetes at 24-h ookinetes with very strong fluorescence levels (Fig. 3). To test IMC1c expression from the paternal allele, parasite line $\mathrm{IMC} 1 \mathrm{c} / \mathrm{mCherry}$ was generated to express a red fluorescent protein-tagged version of $P b I M C 1 c$, which was then crossed with the equivalent GFP-tagged parasite line. The strategy used to generate $\mathrm{IMC} 1 / \mathrm{mCherry}$ was the same as for IMC1c/GFP (Fig. 2). Accordingly, PCR diagnostic for the integration of the selectable marker into the pbimclc locus amplified a $1.8-\mathrm{kb}$ fragment from different clones of this parasite line and not from wild-type parasites, as expected (Fig. 6b). Additionally, PCR diagnostic for the wild-type pbimclc allele amplified a 2.3-kb product from wild-type parasites, but not from the transgenic lines, as expected (Fig. 6b). The resulting $P b \mathrm{IMCl}$ :::mCherry fusion protein displayed similar life-stage expression and subcellular distribution as its GFP-tagged counterpart (data not shown and Fig. 6c). After a genetic cross with parasite line IMC1c/GFP, heterozygous ookinetes (derived from cross fertilization) were produced that dually expressed red and green fluorescent proteins (Fig. 6c). This demonstrates that $P b I M C 1 c$ is expressed from both the maternal- and paternalinherited alleles in the mature ookinete. In a time course, dual expression of GFP and mCherry was first detected at approximately $7 \mathrm{~h}$ post-gametogenesis, indicating this is the point when protein expression from the paternal pbimclc allele commences.

Parasite line IMC1e/GFP exhibited very weak GFP fluorescence in gametocytes (data not shown). In this parasite, fluorescence levels increased around $4 \mathrm{~h}$ post-gametogenesis prior to the start of pellicle formation. To test PbIMC1e expression from the paternal allele, we crossed parasite line IMC1e/GFP with parasite line PbSR/EGFP (Carter et al., 2008). The latter expresses a GFP-tagged version of $P b$ LAP1, which is maternally inherited (Raine et al., 2007). In mature ookinetes, $P b L A P 1$ is almost exclusively present in the crystalloids, which appear as one or two distinctive fluorescent spots in ookinetes of parasite line $\mathrm{PbSR} / \mathrm{EGFP}$ (Carter et al., 2008). In the crossed ookinete culture, we could not detect any mature heterokaryotic ookinetes that displayed, at the same time, fluorescent crystalloids and a fluorescent cortex ( $64 \%$ only peripheral GFP, $36 \%$ only crystalloid GFP; $n=$ 100). A similar result (59\% only peripheral GFP, $41 \%$ only crystalloid GFP; $n=100$ ) was obtained when we crossed IMC1e/GFP with parasite line $P b L A P 3 / G F P$, which expresses a GFP-tagged family member of PbLAP1 that is also maternally expressed (Saeed et al., 2010, 2012). These observations show that, in contrast to PbIMC1c, PbIMC1e is only expressed from the maternal allele in the sexual stages.

$\mathrm{PbIMC1c}$ and PbIMC1e are essential for blood-stage asexual parasite development

To achieve knockout of $P b \mathrm{IMC} 1 \mathrm{c}$ and $P b \mathrm{IMCle}$ expression, we again adopted a strategy of double crossover homologous recombination identical to the GFP-tagging approach. The coding sequences of pbimclc and pbimcle were removed leaving GFP under control of the native pbimcl gene promoters to act as a reporter (Fig. 7a). In contrast to the transfections aimed at GFP tagging, which readily resulted in a specific integration into the pbimclc and pbimcle loci (Fig. 2), our attempts to disrupt pbimclc and pbimcle repeatedly failed to give integration of the selectable marker into the target loci (based on five independent transfections for each gene knockout). This indicated that these genes are important for the development of asexual blood stages and cannot be disrupted, which is consistent with the observed expression of 

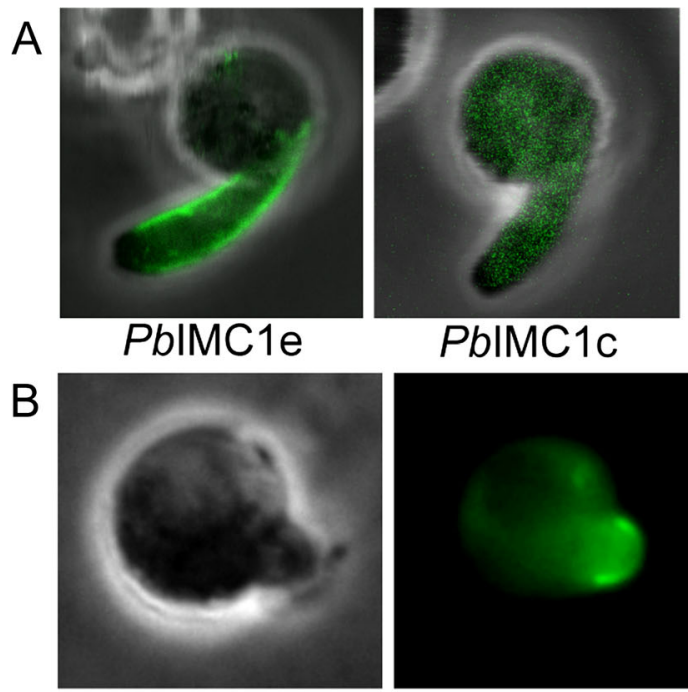

PbIMC1c
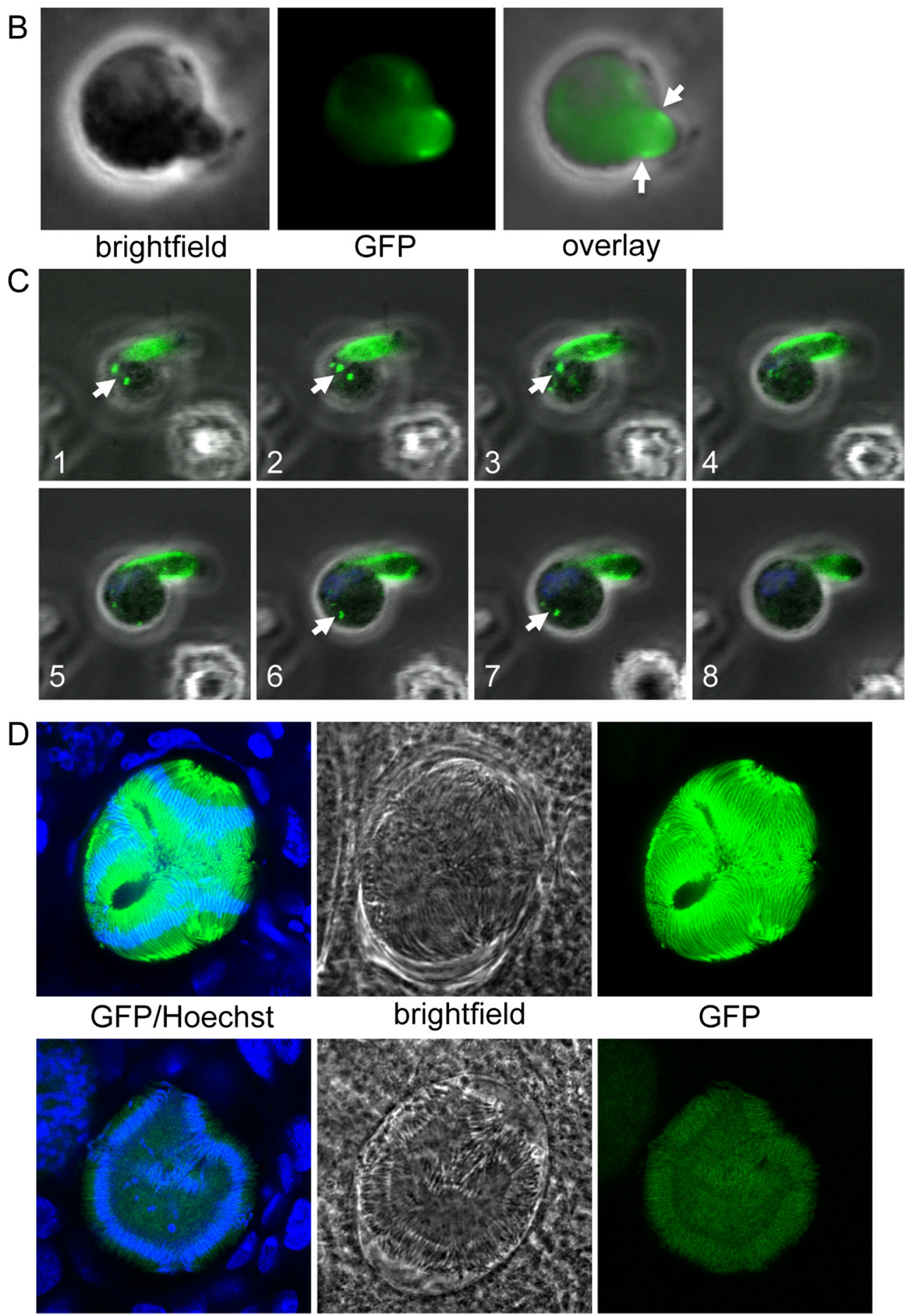

brightfield
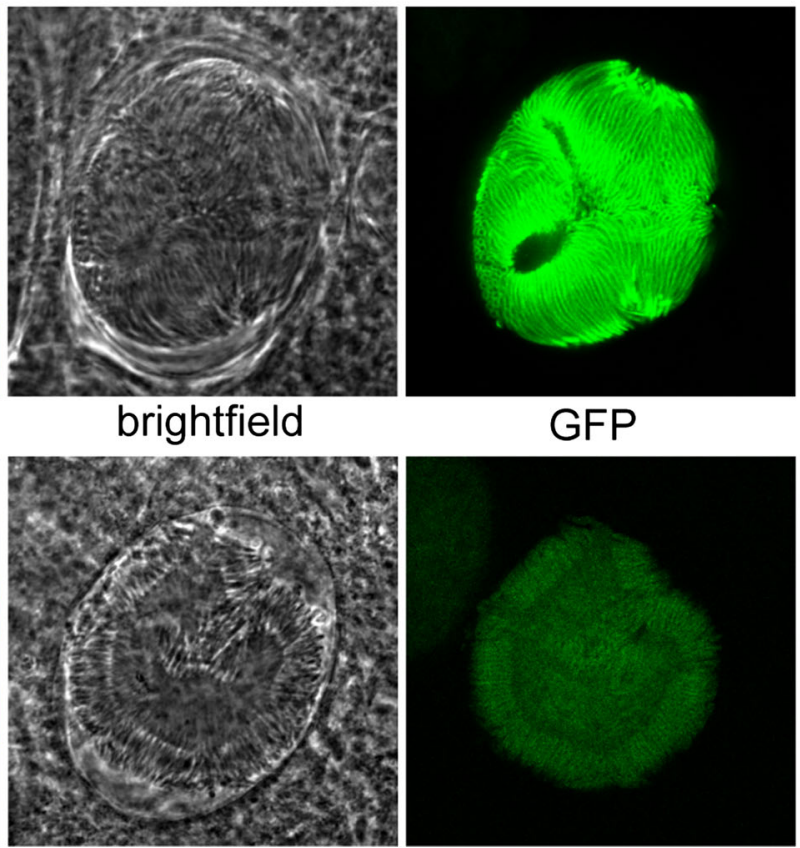

GFP 
Fig. 5 Recruitment of $P b I M C 1$ proteins to the pellicle. a Retort stages at approximately $6 \mathrm{~h}$ post-gametogenesis showing the presence $(\mathrm{PbIMC1 \textrm {e }})$ and absence $(P b \mathrm{IMC} 1 \mathrm{c})$ of pellicular localization. b Very young retort of parasite line IMC1e/GFP at approximately $4 \mathrm{~h}$ post-gametogenesis, exhibiting pellicular localization (arrows). c Serial Z-stack images of a young retort of parasite line IMC1e/GFP, exhibiting fluorescent spots within the spherical part (arrows). d Confocal bright-field and GFP fluorescence images of sporulated oocysts of parasite line IMC1c/GFP, exhibiting strong peripheral fluorescence in the sporozoites (top panels) or very weak cytoplasmic fluorescence (bottom panels). The GFP fluorescence image in the bottom panel was captured using increased photomultiplier gain. The top left hand corner shows part of a not yet sporulated oocyst containing multiple round nuclei (blue) and low-level GFP fluorescence

these genes in asexual blood-stage parasites (Figs. 3 and 4). This notion was corroborated by the fact that the 'knockout' transfections produced drug-resistant parasites that displayed green fluorescence resulting from the expression of the GFP reporter gene. However, in both cases, the GFP fluorescence was only observed in gametocytes, with fluorescence levels being markedly stronger after targeting pbimcle than after targeting pbimclc (Fig. 7b). The lack of integration of the selectable marker and GFP reporter into the target loci, combined with the clear disparity between the expression profiles of GFP in these transfections compared to those that generated GFP-tagged PbIMC1c and PbIMC1e (Figs. 3 and 4), strongly points to integration into non-specific genomic locations. Such events are likely to be selected only when homologous recombination is detrimental to parasite development. Hence, these observations strongly support a critical role for $P b I M C 1 c$ and PbIMC1e in asexual blood-stage development of the parasite.

\section{Discussion}

This study shows that a further two members of the Plasmodium alveolin/IMC1 protein family are recruited to the SPN in the zoite stages where they are expressed as protein. This faithful localization to the pellicle further supports the notion that alveolins have a predominantly cytoskeletal function and, hence, that the structural similarities (i.e. the IMCp domains) reflect functional properties. We show that $P b I M C 1 c$ and $P b I M C 1 e$ are expressed in all three zoite stages of the malaria parasite including merozoites. Recent studies based on cryo-electron tomography failed to detect an apparent subpellicular structure in merozoites, suggesting that a SPN may not be present in this zoite stage (Kudryashev et al., 2012). However, the clear peripheral distribution of $P b I M C 1 c$ in merozoites shown here (Fig. 3) supports the presence of a SPN within the merozoite pellicle. The IMC1 protein expression profiles thus far established by us and others in P. berghei (Khater et al., 2004; Kono et al., 2012;
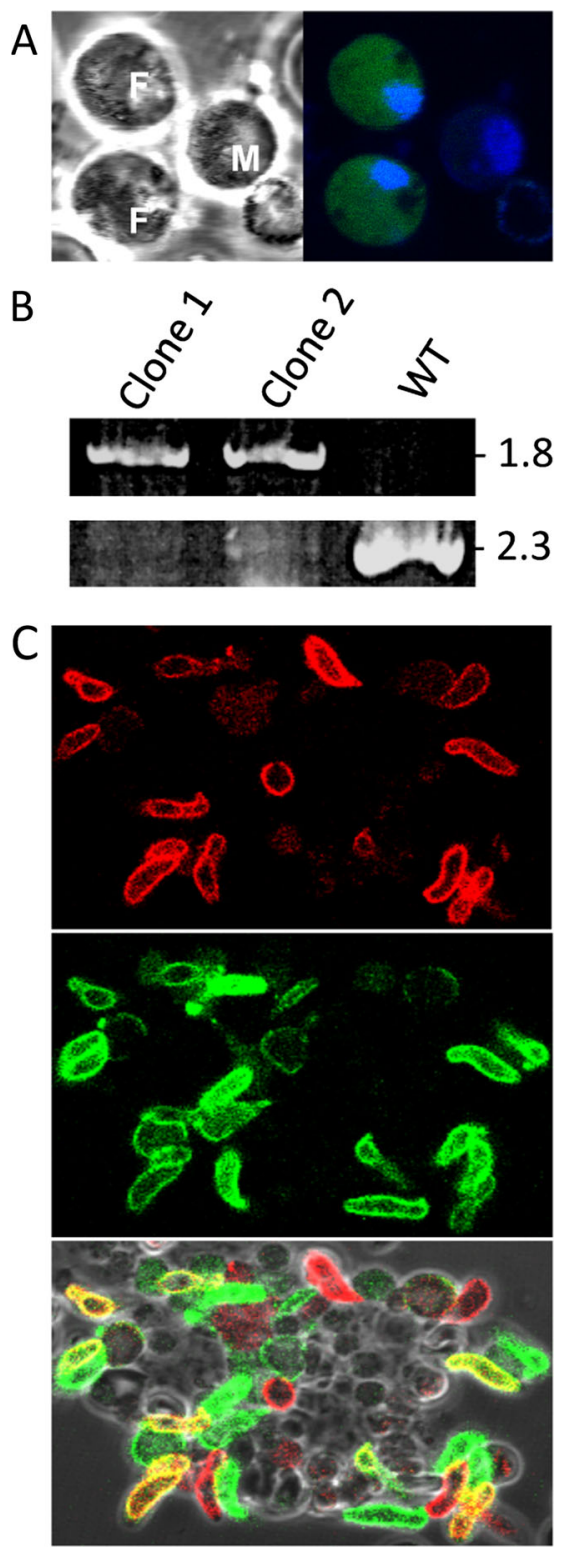

Fig. 6 Expression of $P b I M C 1 c$ from parental alleles in the sexual stages. a Confocal bright-field and GFP fluorescence images of female $(F)$ and male $(M)$ gametocytes of parasite line IMC1c/GFP. Hoechst staining (blue) labels nuclei. b PCR diagnostic for the integration of the selectable marker into the pbimclc locus in two different clones of parasite line $\mathrm{IMC} 1 \mathrm{c} / \mathrm{mCherry}$, amplying a 1.8-kb fragment (top panel). PCR diagnostic for the unmodified pbimclc allele amplified a $2.3-\mathrm{kb}$ product only from WT parasites (bottom panel). c Confocal images of ookinetes derived from a genetic cross between parasite lines IMC1c/GFP and $\mathrm{IMC} 1 \mathrm{c} / \mathrm{mCherry}$, showing mCherry (top) and GFP fluorescence (middle). Bottom panel shows overlay with bright field and identifies dual-labelled ookinetes (yellow) pointing to expression from both parental alleles

Tremp \& Dessens, 2011; Tremp et al., 2008; Volkmann et al., 2012) fit very well with available Plasmodium falciparum protein expression data (Florens et al., 2002; Hall et al., 2005; Lasonder et al., 2002; Lindner et al., 2013; Treeck et al., 2011), indicating that Plasmodium alveolin orthologues 


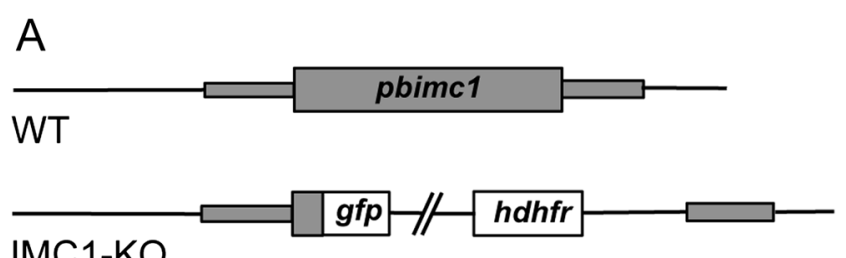

IMC1-KO

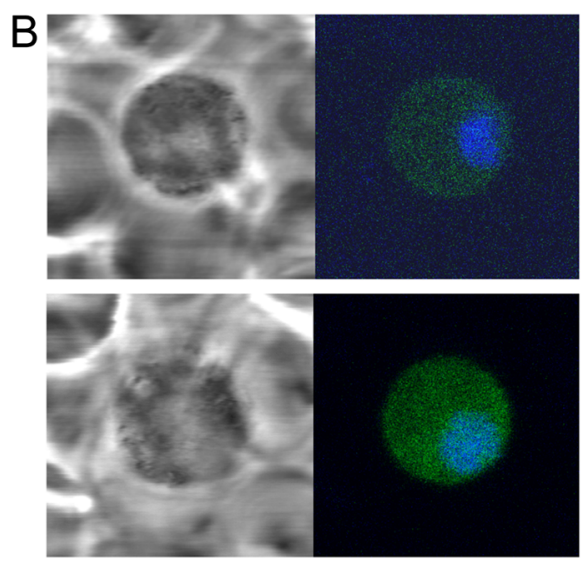

Fig. 7 Targeted disruption of $P b \mathrm{IMCl} \mathrm{c}$ and $P b \mathrm{IMCle}$. a Gene structure of pbimc1 alleles in WT and PbIMC1-KO parasite lines. The pbimc1 gene is indicated with coding sequence (wide bars) and non-coding sequence (narrow bars). Also indicated are the enhanced GFP module $(g f p)$ and the hDHFR selectable marker gene cassette $(h d h f r)$. b Confocal bright-field and GFP fluorescence images of gametocytes after attempted disruption of pbimclc (top panel) and pbimcle (bottom panel). Hoechst DNA staining (blue) labels nuclei

have conserved stage-specific expression profiles. The new alveolin expression data reported here are therefore likely to apply also to $P$. falciparum and other human malaria species. An exception to this may be the gametocyte, which possesses a pellicle structure in $P$. falciparum, but not in $P$. berghei (Dearnley et al., 2012). Moreover, antibodies to a generic alveolin epitope label the periphery of $P$. falciparum gametocytes (Gould et al., 2008), indicating that alveolins are indeed present in the gametocyte SPN.

Our data show for the first time that clear differences exist between Plasmodium alveolins with respect to their assembly into the SPN of ookinetes and sporozoites (Fig. 5). Whereas $P b I M C 1$ e appears to be assembled concurrent with pellicle formation, $P b I M C 1 c$ joins the SPN only after pellicle formation. Accordingly, we anticipate that $P b \mathrm{IMC} 1 \mathrm{c}$ is not required for normal ookinete and sporozoite morphogenesis, in contrast to its family members $P b I M C 1 a, P b I M C 1 b$ and PbIMC1h (Khater et al., 2004; Tremp \& Dessens, 2011; Tremp et al., 2008). Our observations provide a clear demonstration that the SPN continues to develop after zoite formation. This could, for instance, explain why cryo-electron tomography points to midgut sporozoites having a less prominent SPN than salivary gland sporozoites (Kudryashev et al., 2012). Our observations are also consistent with studies of Toxoplasma showing that the SPN of older parasites becomes detergent insoluble, reflecting a change in rigidity and mechanical strength of the structure (Mann et al., 2002). These observations all point to a process of maturation of the SPN after its initial biogenesis.

There are clear parallels between the Plasmodium alveolins described here and some of those characterized in Toxoplasma gondii (Anderson-White et al., 2011). For example, T. gondii IMC1, IMC3, IMC6 and IMC10 localize to the cortical cytoskeleton during tachyzoite daughter cell budding, whereas IMC7, IMC12 and IMC14 are only found in the mature pellicles and not in those of the emerging daughter cells (Anderson-White et al., 2011). Even though the alveolin repertoires differ between Plasmodium and Toxoplasma (Anderson-White et al., 2011; Kono et al., 2012), the distinct chronological assembly of certain family members into the SPN appears to be a common feature that is likely to reflect a biological requirement for different physical properties of the SPN at different phases of zoite development. Another similarity with Toxoplasma alveolins is that, although their main site of action is the cortical cytoskeleton, some are also found in additional subcellular structures such as the basal body and centrosome (Anderson-White et al., 2011). PbIMC1c and $P b I M C 1 e$, too, localize to additional structures (Figs. 3 and 4). For the ookinete, defined basal structures that could correspond to the posterior structure containing $P b I M C 1$ e have not been described. It is notable that the posterior structure associated with the ookinete appears almost exterior of the cell, indicating that it could constitute residual 'zygote' material left over from the transformation of the spherical zygote into the elongated ookinete. Notably, assembly of PbIMCle into the pellicle is accompanied by the formation of multiple discrete PbIMC1e-containing 'spots' that lie mainly within the cytoplasm of the spherical zygote portion, which may become trapped within the residual zygote membrane at the posterior end of the cell. Similar spots were not apparent during the formation of ookinetes that express GFP-tagged PbIMC1b or PbIMC1h (Tremp \& Dessens, 2011; Tremp et al., 2008). In sporozoites, the posterior structure that is labelled with $P b I M C 1 e$ could correspond to, or co-localize with, the posterior polar ring (Kudryashev et al., 2010). It is also not clear what the apical structure in sporozoites labelled with $P b I M C 1 c$ corresponds to. It is notable that the area is present only on one side of the anterior sporozoite, and one possibility is that it could co-localize with the apical ring complex that sits at a sharp angle towards the ventral side of the sporozoite tip (Kudryashev et al., 2012).

Although $\mathrm{PbIMC1c}$ is present in asexual, sexual and sporogonic life stages, it is not constitutively expressed as the protein was not detected in male gametocytes or in oocysts before sporulation. The apparent lack of GFP fluorescence in male gametocytes indicates that $P b \mathrm{IMCl} c$ is not carried over from the preceding trophozoite stage; if this was the case, both male and female gametocytes would be expected to express GFP. Rather, the restricted expression in female gametocytes 
points to an early commitment to sexual stage development that occurs before trophozoite development. Female gametocytes in P. berghei are spherical cells that do not possess a discernible pellicle, so it is not clear why the protein is expressed here. One possibility is that PbIMC1c could have a function in the gametocyte that is not linked to the SPN. It should be noted that a study by Mair and colleagues (Mair et al., 2006) shows significantly reduced transcript levels of both pbimclc and pblmcle in gametocytes of the helicase DOZI (development of zygote inhibited) null mutant parasites. This suggests that these genes are subject to translational repression, a female gametocyte-specific mechanism of translational silencing involved in the development of the parasite post-fertilization (Mair et al., 2006). Translational repression of pbimclc and pbimcle is consistent with the failure to detect significant amounts of the respective gene products in gametocytes using high-accuracy mass spectrometry-based proteomics (Hall et al., 2005; Khan et al., 2005). In P. falciparum, too, pfimclc and pfimcle mRNAs are abundant in mature blood-stage gametocytes (Lopez-Barragan et al., 2011), while the corresponding gene products have not been detected in this life stage by mass spectrometry (Silvestrini et al., 2010), again supporting a scenario of translational repression. The low expression of PbIMC1c observed in female gametocytes could be the result of 'leaky' translational repression, where only a fraction of the pbimclc mRNA is silenced.

The failure to achieve a structural disruption of the pbimclc and pbimcle genes indicates that these genes are refractory to genetic depletion. This, in turn, indicates that these genes are essential for the completion of the cycle of blood-stage schizogony or for infectivity of the merozoites. Besides repeated failure of the transfections aimed at gene 'knockout' in contrast to those aimed at gene 'tagging', we obtained additional evidence which strongly supports a vital role of $P b \mathrm{IMC} 1 \mathrm{c}$ and $P b I M C 1 e$ in blood-stage parasite development: In both cases the transfections aimed at gene disruption resulted in a nonspecific integration of the GFP reporter and the accompanying drug selection marker, giving rise to green fluorescent gametocytes (Fig. 7b). We presume that these events must have occurred via non-homologous recombination-based integration into a 'random' gene, leaving its respective promoter to drive GFP reporter expression. Because homologous recombination is much more efficient than non-homologous recombination, the latter is likely to be selected only when homologous recombination is detrimental to parasite development. Interestingly, we obtained similar GFP expression after replicate transfections, suggesting that the integration site may not be entirely indiscriminate and perhaps could constitute the 'next best' site with regards to sequence homology with the target DNA. We propose that this phenomenon can be a useful marker for the identification of genes that are vital for asexual development.
Acknowledgments This work was supported by the Wellcome Trust, grants 076648 and 088449 , and a Studentship to FSA-K from the Cultural Bureau of the Royal Embassy of Saudi Arabia in London. We thank E McCarthy for the assistance with microscopy.

Conflict of interest The authors declare that they have no conflict of interest.

Open Access This article is distributed under the terms of the Creative Commons Attribution License which permits any use, distribution, and reproduction in any medium, provided the original author(s) and the source are credited.

\section{References}

Anderson-White BR, Ivey FD, Cheng K, Szatanek T, Lorestani A, Beckers CJ, Ferguson DJ, Sahoo N, Gubbels MJ (2011) A family of intermediate filament-like proteins is sequentially assembled into the cytoskeleton of Toxoplasma gondii. Cell Microbiol 13(1):18-31

Arai M, Billker O, Morris HR, Panico M, Delcroix M, Dixon D, Ley SV, Sinden RE (2001) Both mosquito-derived xanthurenic acid and a host blood-derived factor regulate gametogenesis of Plasmodium in the midgut of the mosquito. Mol Biochem Parasitol 116(1):17-24

Bannister LH, Hopkins JM, Fowler RE, Krishna S, Mitchell GH (2000) A brief illustrated guide to the ultrastructure of Plasmodium falciparum asexual blood stages. Parasitol Today 16(10):427-433

Carter V, Shimizu S, Arai M, Dessens JT (2008) PbSR is synthesized in macrogametocytes and involved in formation of the malaria crystalloids. Mol Microbiol 68(6):1560-1569

Dearnley MK, Yeoman JA, Hanssen E, Kenny S, Turnbull L, Whitchurch CB, Tilley L, Dixon MW (2012) Origin, composition, organization and function of the inner membrane complex of Plasmodium falciparum gametocytes. J Cell Sci 125(8):2053-2063

Dessens JT, Beetsma AL, Dimopoulos G, Wengelnik K, Crisanti A, Kafatos FC, Sinden RE (1999) CTRP is essential for mosquito infection by malaria ookinetes. EMBO J 18(22):6221-6227

Florens L, Washburn MP, Raine JD, Anthony RM, Grainger M, Haynes JD, Moch JK, Muster N, Sacci JB, Tabb DL, Witney AA, Wolters D, Wu Y, Gardner MJ, Holder AA, Sinden RE, Yates JR, Carucci DJ (2002) A proteomic view of the Plasmodium falciparum life cycle. Nature 419(6906):520-526

Gould SB, Tham WH, Cowman AF, McFadden GI, Waller RF (2008) Alveolins, a new family of cortical proteins that define the protist infrakingdom Alveolata. Mol Biol Evol 25(6):1219-1230

Hall N, Karras M, Raine JD, Carlton JM, Kooij TW, Berriman M, Florens L, Janssen CS, Pain A, Christophides GK, James K, Rutherford K, Harris B, Harris D, Churcher C, Quail MA, Ormond D, Doggett J, Trueman HE, Mendoza J, Bidwell SL, Rajandream MA, Carucci DJ, Yates JR 3rd, Kafatos FC, Janse CJ, Barrell B, Turner CM, Waters AP, Sinden RE (2005) A comprehensive survey of the Plasmodium life cycle by genomic, transcriptomic, and proteomic analyses. Science 307(5706):82-86

Khan SM, Franke-Fayard B, Mair GR, Lasonder E, Janse CJ, Mann M, Waters AP (2005) Proteome analysis of separated male and female gametocytes reveals novel sex-specific Plasmodium biology. Cell 121(5):675-687

Khater EI, Sinden RE, Dessens JT (2004) A malaria membrane skeletal protein is essential for normal morphogenesis, motility, and infectivity of sporozoites. J Cell Biol 167(3):425-432

Kono M, Herrmann S, Loughran NB, Cabrera A, Engelberg K, Lehmann C, Sinha D, Prinz B, Ruch U, Heussler V, Spielmann T, Parkinson J, Gilberger TW (2012) Evolution and architecture of the inner 
membrane complex in asexual and sexual stages of the malaria parasite. Mol Biol Evol 29(9):2113-2132

Kudryashev M, Lepper S, Stanway R, Bohn S, Baumeister W, Cyrklaff M, Frischknecht F (2010) Positioning of large organelles by a membrane- associated cytoskeleton in Plasmodium sporozoites. Cell Microbiol 12(3):362-371

Kudryashev M, Munter S, Lemgruber L, Montagna G, Stahlberg H, Matuschewski K, Meissner M, Cyrklaff M, Frischknecht F (2012) Structural basis for chirality and directional motility of Plasmodium sporozoites. Cell Microbiol 14(11):1757-1768

Lasonder E, Ishihama Y, Andersen JS, Vermunt AM, Pain A, Sauerwein RW, Eling WM, Hall N, Waters AP, Stunnenberg HG, Mann M (2002) Analysis of the Plasmodium falciparum proteome by highaccuracy mass spectrometry. Nature 419(6906):537-542

Lindner SE, Swearingen KE, Harupa A, Vaughan AM, Sinnis P, Moritz RL, Kappe SH (2013) Total and putative surface proteomics of malaria parasite salivary gland sporozoites. Mol Cell Proteomics 12(5):1127-1143

Lopez-Barragan MJ, Lemieux J, Quinones M, Williamson KC, MolinaCruz A, Cui K, Barillas-Mury C, Zhao K, Su XZ (2011) Directional gene expression and antisense transcripts in sexual and asexual stages of Plasmodium falciparum. BMC Genomics 12:587

Mair GR, Braks JA, Garver LS, Wiegant JC, Hall N, Dirks RW, Khan SM, Dimopoulos G, Janse CJ, Waters AP (2006) Regulation of sexual development of Plasmodium by translational repression. Science 313(5787):667-669

Mann T, Beckers C (2001) Characterization of the subpellicular network, a filamentous membrane skeletal component in the parasite Toxoplasma gondii. Mol Biochem Parasitol 115(2):257-268

Mann T, Gaskins E, Beckers C (2002) Proteolytic processing of TgIMC1 during maturation of the membrane skeleton of Toxoplasma gondii. J Biol Chem 277(43):41240-41246

Meis JF, Ponnudurai T (1987) Ultrastructural studies on the interaction of Plasmodium falciparum ookinetes with the midgut epithelium of Anopheles stephensi mosquitoes. Parasitol Res 73(6):500-506

Meis JF, Pool G, van Gemert GJ, Lensen AH, Ponnudurai T, Meuwissen JH (1989) Plasmodium falciparum ookinetes migrate intercellularly through Anopheles stephensi midgut epithelium. Parasitol Res 76(1):13-19

Morrissette NS, Sibley LD (2002) Cytoskeleton of apicomplexan parasites. Microbiol Mol Biol Rev 66(1):21-38
Raine JD, Ecker A, Mendoza J, Tewari R, Stanway RR, Sinden RE (2007) Female inheritance of malarial lap genes is essential for mosquito transmission. PLoS Pathog 3(3):e30

Saeed S, Carter V, Tremp AZ, Dessens JT (2010) Plasmodium berghei crystalloids contain multiple LCCL proteins. Mol Biochem Parasitol 170(1):49-53

Saeed S, Tremp AZ, Dessens JT (2012) Conformational codependence between Plasmodium berghei LCCL proteins promotes complex formation and stability. Mol Biochem Parasitol 185(2):170-173

Santos JM, Lebrun M, Daher W, Soldati D, Dubremetz JF (2009) Apicomplexan cytoskeleton and motors: key regulators in morphogenesis, cell division, transport and motility. Int J Parasitol 39(2): $153-162$

Silvestrini F, Lasonder E, Olivieri A, Camarda G, van Schaijk B, Sanchez M, Younis Younis S, Sauerwein R, Alano P (2010) Protein export marks the early phase of gametocytogenesis of the human malaria parasite Plasmodium falciparum. Mol Cell Proteomics 9(7):14371448

Treeck M, Sanders JL, Elias JE, Boothroyd JC (2011) The phosphoproteomes of Plasmodium falciparum and Toxoplasma gondii reveal unusual adaptations within and beyond the parasites' boundaries. Cell Host Microbe 10(4): $410-419$

Tremp AZ, Dessens JT (2011) Malaria IMC1 membrane skeleton proteins operate autonomously and participate in motility independently of cell shape. J Biol Chem 286(7):5383-5391

Tremp AZ, Khater EI, Dessens JT (2008) IMC1b is a putative membrane skeleton protein involved in cell shape, mechanical strength, motility, and infectivity of malaria ookinetes. J Biol Chem 283(41): 27604-27611

Tremp AZ, Carter V, Saeed S, Dessens JT (2013) Morphogenesis of Plasmodium zoites is uncoupled from tensile strength. Mol Microbiol 89(3):552-564

Volkmann K, Pfander C, Burstroem C, Ahras M, Goulding D, Rayner JC, Frischknecht F, Billker O, Brochet M (2012) The alveolin IMC1h is required for normal ookinete and sporozoite motility behaviour and host colonisation in Plasmodium berghei. PLoS One 7(7):e41409

Waters AP, Thomas AW, van Dijk MR, Janse CJ (1997) Transfection of malaria parasites. Methods 13(2):134-147 